\title{
Phenylephrine increases cardiac output by raising cardiac preload in patients with anesthesia induced hypotension
}

\author{
A. F. Kalmar ${ }^{1,3}$ - S. Allaert ${ }^{1} \cdot$ P. Pletinckx ${ }^{2} \cdot$ J.-W. Maes ${ }^{1} \cdot$ J. Heerman ${ }^{1} \cdot$ J. J. Vos ${ }^{3} \cdot$ M. M. R. F. Struys $s^{3,4}$. \\ T. W. L. Scheeren ${ }^{3}$
}

Received: 23 November 2017 / Accepted: 7 March 2018 / Published online: 22 March 2018

(c) The Author(s) 2018

\begin{abstract}
Induction of general anesthesia frequently induces arterial hypotension, which is often treated with a vasopressor, such as phenylephrine. As a pure $\alpha$-agonist, phenylephrine is conventionally considered to solely induce arterial vasoconstriction and thus increase cardiac afterload but not cardiac preload. In specific circumstances, however, phenylephrine may also contribute to an increase in venous return and thus cardiac output (CO). The aim of this study is to describe the initial time course of the effects of phenylephrine on various hemodynamic variables and to evaluate the ability of advanced hemodynamic monitoring to quantify these changes through different hemodynamic variables. In 24 patients, after induction of anesthesia, during the period before surgical stimulus, phenylephrine $2 \mu \mathrm{g} \mathrm{kg}^{-1}$ was administered when the MAP dropped below $80 \%$ of the awake state baseline value for $>3 \mathrm{~min}$. The mean arterial blood pressure (MAP), heart rate (HR), end-tidal $\mathrm{CO}_{2}$ $\left(\mathrm{EtCO}_{2}\right)$, central venous pressure (CVP), stroke volume (SV), CO, pulse pressure variation (PPV), stroke volume variation (SVV) and systemic vascular resistance (SVR) were recorded continuously. The values at the moment before administration of phenylephrine and $5\left(\mathrm{~T}_{5}\right)$ and $10\left(\mathrm{~T}_{10}\right)$ min thereafter were compared. After phenylephrine, the mean(SD) MAP, SV, $\mathrm{CO}, \mathrm{CVP}$ and $\mathrm{EtCO}_{2}$ increased by 34(13) $\mathrm{mmHg}, 11(9) \mathrm{mL}, 1.02(0.74) \mathrm{L} \mathrm{min}^{-1}, 3(2.6) \mathrm{mmHg}$ and $4.0(1.6) \mathrm{mmHg}$ at $\mathrm{T}_{5}$ respectively, while both dynamic preload variables decreased: PPV dropped from $20 \%$ at baseline to $9 \%$ at $\mathrm{T}_{5}$ and to $13 \%$ at $\mathrm{T}_{10}$ and SVV from 19 to 11 and 14\%, respectively. Initially, the increase in MAP was perfectly aligned with the increase in SVR, until $150 \mathrm{~s}$ after the initial increase in MAP, when both curves started to dissociate. The dissociation of the evolution of MAP and SVR, together with the changes in PPV, CVP, $\mathrm{EtCO}_{2}$ and $\mathrm{CO}$ indicate that in patients with anesthesia-induced hypotension, phenylephrine increases the $\mathrm{CO}$ by virtue of an increase in cardiac preload.
\end{abstract}

Keywords Hemodynamic monitoring $\cdot$ Fluid responsiveness $\cdot$ Phenylephrine $\cdot$ Cardiac output $\cdot$ Pulse pressure variation

\section{Introduction}

The ultimate goal of hemodynamic management is to maintain adequate tissue oxygen delivery to the different end-organs [1]. Surgical patients often suffer relative hypo-

A. F. Kalmar

Alainkalmar@gmail.com

1 Department of Anesthesia and Critical Care Medicine, Maria Middelares Hospital, Buitenring Sint-Denijs 30, 9000 Ghent, Belgium

2 Department of Surgery, Maria Middelares Hospital, Ghent, Belgium

3 Department of Anesthesiology, University of Groningen, University Medical Center Groningen, Groningen, The Netherlands

4 Department of Anesthesia, Ghent University, Ghent, Belgium volemia owing to a combination of epidural analgesia, general anesthesia and patient positioning. The principal aim of goal-directed fluid therapy is to optimize the position of the heart on the Frank-Starling curve by increasing cardiac preload. This is conventionally pursued by administration of fluids to increase total blood volume and can improve patient outcome by reducing postoperative complications and length of hospital stay [2].

Phenylephrine is a direct $\alpha$-adrenergic receptor agonist, predominantly $\alpha_{1}$, increasing the systemic vascular resistance (SVR) and arterial pressure [3]. While venous 
$\alpha_{1}$ receptor activity is acknowledged scientifically, in most clinical conditions, phenylephrine is considered to increase cardiac afterload but not cardiac preload. In patients with life threatening septic shock, norepinephrine has been shown to increase venous return in case of preload dependence $[4,5]$. Nevertheless, while a beneficial effect of phenylephrine on the blood pressure is obviously well known, phenylephrine is conventionally postulated to have no effects on $\mathrm{CO}$, but owing to an increase in afterload, would in most cases even decrease CO. In contrast, however, while an increase in left ventricular afterload may decrease stroke volume (SV) and thus cardiac output (CO) [6], the $\alpha_{1}$-adrenergic receptor stimulation-either by phenylephrine or norepinephrinealso decreases venous capacitance, which could in turn increase cardiac preload and SV [3]. It has been shown in pigs that the impact of phenylephrine on the $\mathrm{CO}$ is related to preload dependency [7]. When the heart is preload independent, phenylephrine induces on average a decrease in $\mathrm{CO}$, whereas when the heart is preload dependent, it induces on average an increase in CO [6]. We hypothesize that in preload-dependent patients due to pronounced relative hypovolemia induced by combined epidural and general anesthesia, in leg-down position, phenylephrine may increase cardiac preload by virtue of centralisation of blood volume.

The aim of this study was to differentiate the chronicity of the changes in cardiac preload and $\mathrm{CO}$ after a single administration of phenylephrine and to assess the ability of advanced hemodynamic monitoring to quantify these changes through different hemodynamic variables.

\section{Methods}

This prospective interventional study was approved by the institutional review board and was registered at clinicaltrials.gov (NCT:02739399; PI Dr. A Kalmar; April 15, 2016). The manuscript adheres to the applicable STROBE guidelines. After written informed consent was obtained, a total of 24 adult patients, scheduled for elective laparoscopic sigmoidectomy were included (Fig. 1). Patients with cardiac arrhythmia or a contraindication for atropine or phenylephrine administration were excluded.

\subsection{Study protocol}

No premedication was administered. Upon arrival in the operating theatre, a peripheral intravenous line was inserted and an epidural catheter was placed. After adequate preoxygenation, induction and maintenance of anesthesia was pursued by target-controlled total i.v. anesthesia with propofol and remifentanil. At the start of induction of anesthesia, intravenous methylatropine $0.5 \mathrm{mg}$ and epidural levobupivacaine $50 \mathrm{mg}$ were given. After the administration
Assessed for eligibility $(n=27)$

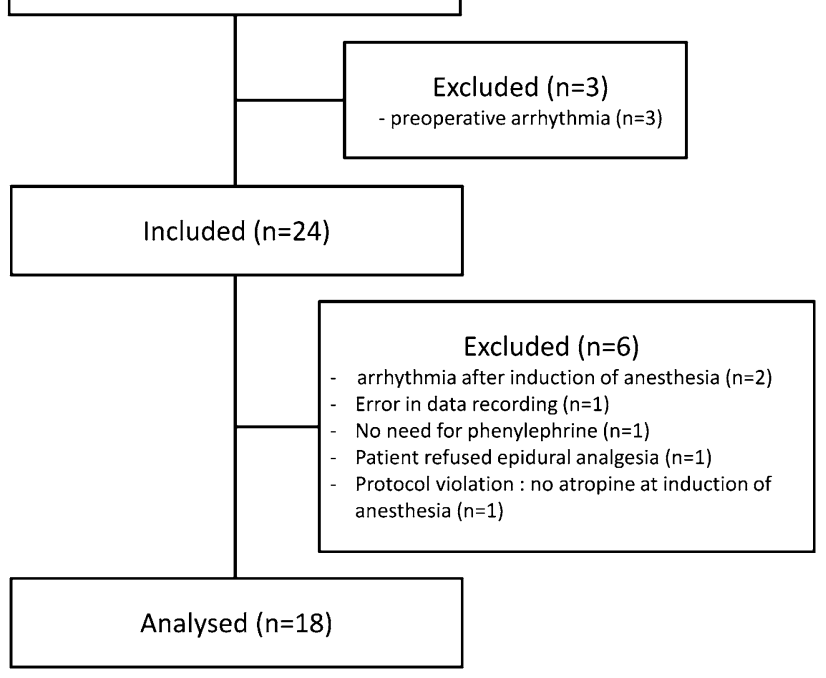

Fig. 1 Flow chart of the patients' inclusion and analysis

of cis-atracurium and endotracheal intubation, the patients' lungs were mechanically ventilated in the volume control mode (tidal volume: $8 \mathrm{~mL} \mathrm{~kg}^{-1}$ ) with an $\mathrm{O}_{2}$ /air mixture $\left(\mathrm{F}_{\mathrm{i}} \mathrm{O}_{2}\right.$ 0.6) and a PEEP of $4 \mathrm{~cm} \mathrm{H}_{2} \mathrm{O}$. During the study period, the ventilatory settings were unchanged. A radial artery was cannulated using a $20 \mathrm{G}$ catheter and connected with a disposable ProAQT transducer from the Pulsioflex monitor (Maquet, Rastatt, Germany) —all measurements were conducted with the same Pulsioflex monitor and automatically calibrated. This minimal invasive device enables calculation of the mean arterial blood pressure (MAP), heart rate (HR), SV and CO using pulse contour analysis of the arterial pressure curve. In addition, it calculates pulse pressure variation (PPV) and stroke volume variation (SVV) as measures of cardiac preload dependency as well as SVR as one of the determinants of left ventricular afterload. Next, a central venous catheter was placed for continuous recording of the central venous pressure (CVP) and the patient was positioned in preparation for surgery. All pressure transducers were located at the level of the right atrium before initiation of the study period. During the subsequent period prior to surgical stimulus, when MAP dropped below $80 \%$ of the awake state baseline value for $>3 \mathrm{~min}$, a bolus of phenylephrine $2 \mu \mathrm{g} \mathrm{kg}^{-1}$ was administered. From 3 min before until 13 min after phenylephrine administration, patient positioning was left unaltered, and no other medication or fluid was administered.

\subsection{Data registration and analysis}

All anesthetic data were collected on the anesthesia monitor (Philips MP70; Philips, Eindhoven, The Netherlands) 
and recorded at $0.2 \mathrm{~Hz}$ for subsequent offline analysis. The electronic data were imported into Microsoft Excel 2010® (Microsoft, Redmond, USA) for analysis. In addition to the recorded variables, an analogue of the mean systemic filling pressure (Pmsa) was calculated using the formula Pms $\mathrm{a}=\mathrm{a} \times \mathrm{CVP}+\mathrm{b} \times \mathrm{MAP}+\mathrm{c} \times \mathrm{CO}$, in which $\mathrm{a}=0.96, \mathrm{~b}=0.04$ and $\mathrm{c}$ is calculated according to anthropometric data [8]. Subsequently, resistance to venous return (RVR) was calculated as $\mathrm{RVR}=(\mathrm{Pmsa}-\mathrm{CVP}) . \mathrm{CO}^{-1}$ and pressure for venous return (Pvr; as a measure of venous return) was calculated as $\mathrm{Pvr}=$ Pmsa - CVP.

The evolution of the absolute values and of the changes relative to baseline $\left(T_{-1}\right)$ was analyzed from 1 min before induction $\left(\mathrm{T}_{-1}\right)$ of anesthesia until the relative steady state was achieved for all study variables after $10 \min \left(\mathrm{T}_{10}\right)$. All measurements for the study were performed before surgery commenced.

\subsection{Statistical analysis}

Assuming a normal distribution of the $\mathrm{CO}$ data, we considered a mean increase in $\mathrm{CO}$ of $15 \%$ to be clinically relevant (estimated SD of $0.7 \mathrm{~L} \mathrm{~min}^{-1}$, based on pilot data). To detect this difference with an $\alpha$-error of 0.05 and a power of 0.95 , a total of 17 patients is needed [9]. A supplemental $40 \%$ of patients were included to anticipate exclusions, making a total of 24 patients.

Normality and homoscedasticity were tested with the Kolmogorov-Smirnov test and modified Levine test, respectively. Continuous data are expressed as mean(SD). For statistical analysis and visualization, the individual patient measurements were synchronized at the moment $\left(\mathrm{T}_{0}\right)$ of $10 \mathrm{mmHg}$ increase in MAP after phenylephrine administration.

For visual assessment of systematic changes of the main variables, the evolution of the individual patient values, as well as the evolution of the mean value were depicted in Fig. 2. For comprehensive visualization of the chronicity and interaction of the different variables, the average values of all the studied variables were shown in Fig. 3.

The absolute values of the analysed variables were determined at 1 min before the increase in MAP $\left(\mathrm{T}_{-1}\right)$, and $5\left(\mathrm{~T}_{5}\right)$ and $10\left(\mathrm{~T}_{10}\right)$ min afterwards. Results were subject to the general linear model repeated measures ANOVA with Bonferroni adjustment. All statistics were performed using S-PLUS 8.0 (TIBCO Software Inc., Palo Alto, CA, USA) and SPSS 23.0 (SPSS Inc., Chicago, IL, USA). Significance was set at $\mathrm{P}<0.05$.

\section{Results}

Six patients were excluded from analysis because of predetermined exclusion criteria: arrhythmia $(n=2)$, technical error, or absence of epidural analgesia, atropine, phenylephrine need (Fig. 1). A total of 18 ASA 2-3 patients were included in the analysis (Fig. 1). The mean(SD) age was 62(13) years, the weight was 74(12) $\mathrm{kg}$, and the length was $165(7) \mathrm{cm}$.

The average(SD) CO increased from 3.92(0.87) $\mathrm{L} \mathrm{min}^{-1}$ at $\mathrm{T}_{-1}$ to $4.94(1.2) \mathrm{L} \mathrm{min}^{-1}$ at $\mathrm{T}_{5}$. Figure $2 \mathrm{a}-\mathrm{k}$ shows the evolution in individual patients (thin lines) and average (thick line) values of the main hemodynamic variables during the period from $1 \mathrm{~min}$ before till $12 \mathrm{~min}$ after the increase in initial blood pressure. An overview of the chronicity and interactions of the mean values of all the investigated variables is comprehensively depicted in Fig. 3.

Changes in hemodynamics are summarized in Table 1. Between $\mathrm{T}_{-1}$ and $\mathrm{T}_{5}, \mathrm{MAP}, \mathrm{SV}, \mathrm{CO}, \mathrm{CVP}$ and $\mathrm{EtCO}_{2}$ increased by $62,28,26,33$ and $11 \%$, respectively, while both dynamic preload variables decreased: PPV dropped from $20 \%$ at $\mathrm{T}_{-1}$ to $9 \%$ at $\mathrm{T}_{5}$ and to $13 \%$ at $\mathrm{T}_{10}$ and $\mathrm{SVV}$ from 19 to 11 and $14 \%$, respectively. Between $\mathrm{T}_{-1}$ and $\mathrm{T}_{5}$, the Pmsa increased by $37 \%$ and the Pvr by $41 \%$. In addition, while the SVR increased by $37 \%$, the RVR increased by $6 \%$ only.

\section{Discussion}

Phenylephrine is conventionally thought to negatively affect $\mathrm{CO}$, or at best to have no influence if the cardiac contractility is able to overcome the increased afterload without loss of SV [10]. Our hypothesis, however, was on the contrary, that if a relative hypovolemia is present due to anesthesia-induced excessive vasodilation of the capacitance vessels, this could be corrected by phenylephrine, inducing an improved centralization of the available blood, eventually resulting in an increase in CO.

The main finding of this prospective study was that in patients with anesthesia-induced hypotension and preload dependency-defined as PPV $>12 \%$, phenylephrine increases $\mathrm{CO}$ by virtue of an increase in return function. This is reflected in multiple distinct indices, all indicating an increase in $\mathrm{CO}$, owing to a rightward shift in the position of the heart on the Frank-Starling relationship: the dissociation of MAP and SVR at $\mathrm{T}_{1}, \mathrm{CVP}, \mathrm{PPV}, \mathrm{CO}$, and $\mathrm{EtCO}_{2}$.

In patients undergoing sigmoidectomy, a relative hypovolemia is common owing to the combination of several factors: the patients have been fasting from the night before, had bowel preparation, and received an epidural loading dose, combined with general anesthesia, all causing vasodilation. 


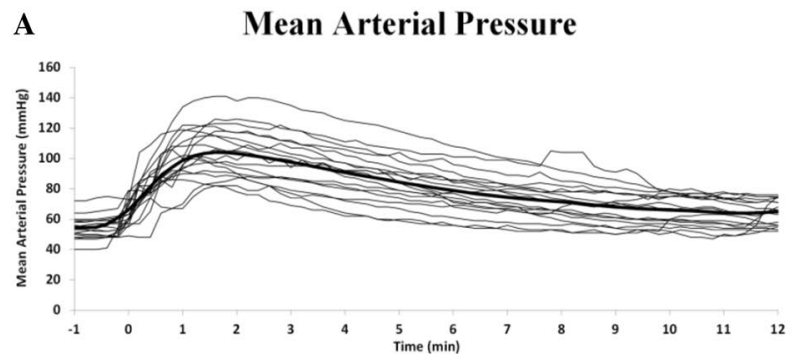

C

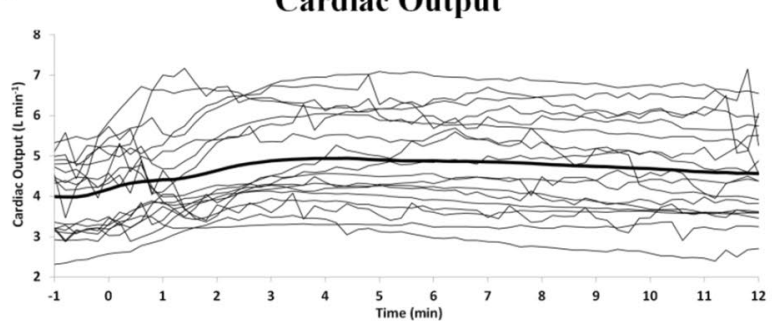

E

End-Tidal $\mathrm{CO}_{2}$

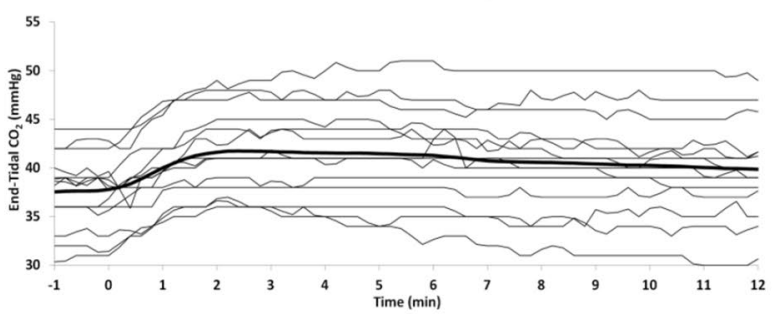

G

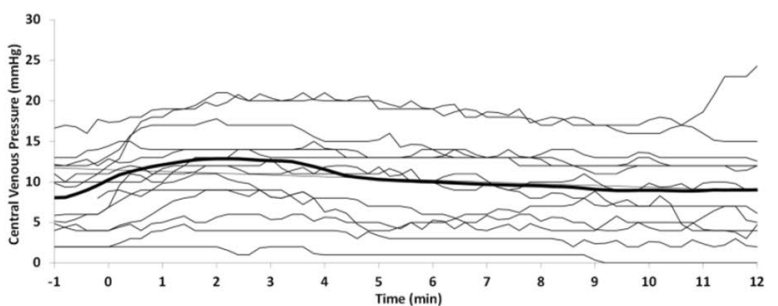

I

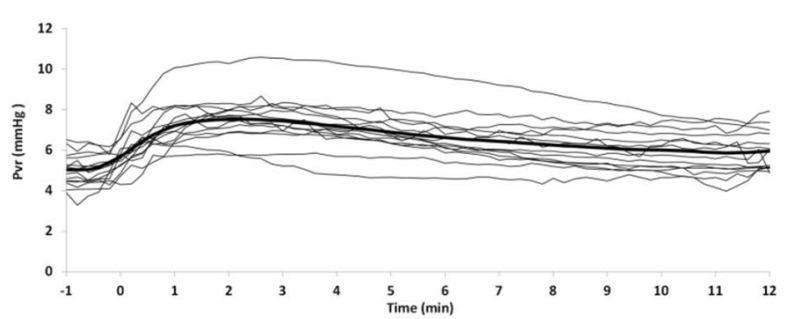

K

Stroke Volume Variation

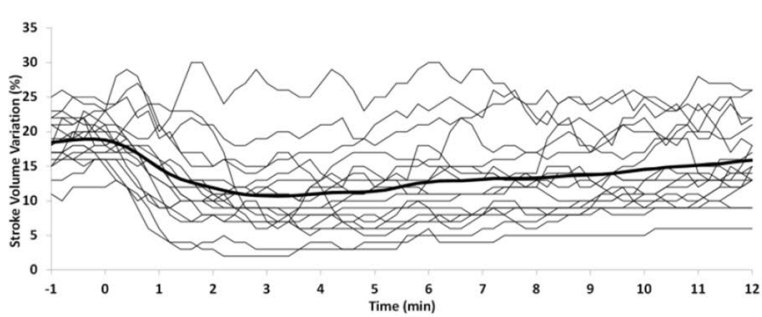

B Systemic Vascular Resistance

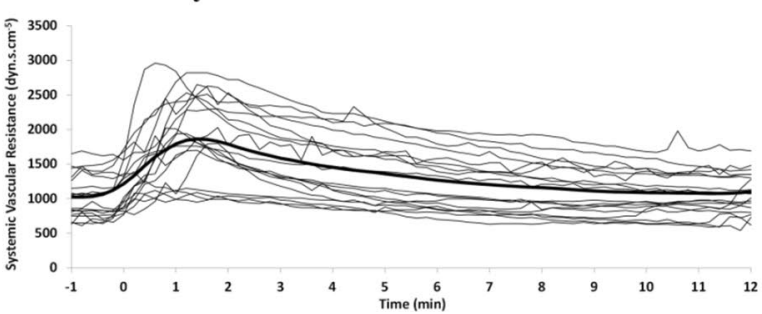

D Stroke Volume
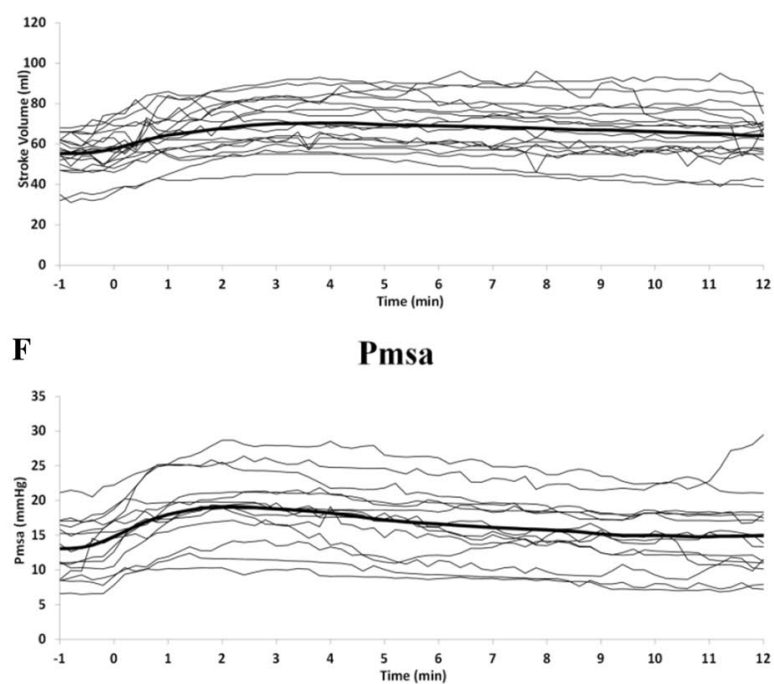

H RVR

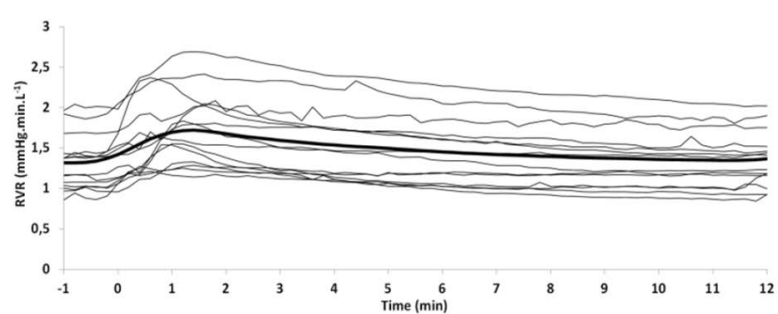

J Pulse Pressure Variation

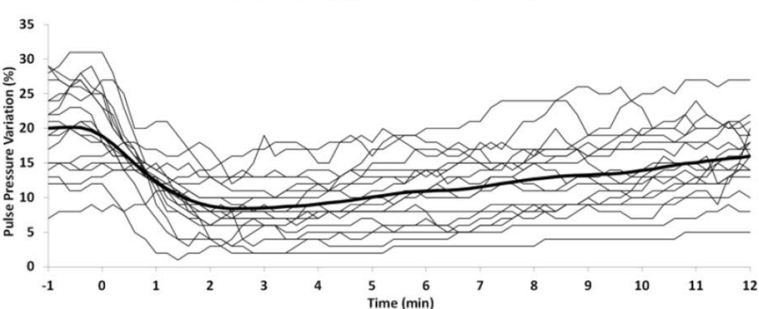


4Fig. 2 The evolution of individual patient variables. The evolution in individual patients (thin lines) and average (thick line) values of the main preload-dependent variables over the period from 1 min before till $12 \mathrm{~min}$ after the increase in initial blood pressure following the administration of phenylephrine. All measurements are synchronized at the moment $\left(\mathrm{T}_{0}\right)$ of $10 \mathrm{mmHg}$ increase in MAP following phenylephrine administration

This pharmacologically induced vasoplegia, together with gravitational venous pooling in the lower limbs due to the leg-down patient positioning - to prevent conflict with the surgeon's arms - and an increase in abdominal pressure often results in a markedly decreased venous return [11]. While an increase in cardiac preload enhances the $\mathrm{CO}$, recent evidence revealed that a zero-balance fluid approach is recommended in the elective perioperative setting to avoid adverse effects of unnecessary, excessive fluid administration [12, 13]. In bowel surgery, the additional concern for return of peristalsis, and oedema of the gut tissue emphasizes the importance of moderating fluid administration. Fluid restriction is therefore considered a part of the care package for enhanced recovery after colorectal surgery [14].

Importantly, this relative hypovolemic state is just of temporary occurrence. When surgery ends, and the patient awakens, the venous tonus and consequently $\mathrm{CO}$ will increase again spontaneously. As such, an alternative to enhance venous return, other than the irrevocable administration of fluids would be desirable. In those cases, the option of recruiting internal blood volume by increasing venous return through reversible pharmacological means such as vasopressors acting predominantly on capacitance vessels might be a potential alternative.

In an animal study, the average effect of phenylephrine on $\mathrm{CO}$ was related to the preload dependency of the heart: when the heart was preload dependent, phenylephrine induced an increase in CO [7]. Similarly, in a study with human patients, CO and SV decreased in preload-independent patients through an increase in cardiac afterload, but by virtue of increased venous return were unchanged in those that were preload-dependent [6].

In contrast to most studies analysing the combined effects of phenylephrine [3], its beneficial effect on the return function is much more pronounced in our patients where the combination of general anesthesia, leg-down position and epidural analgesia - which reduces alpha tone — induced distinctly different physiological conditions [15]. The actual blood flow in the body is determined by the intersection of the cardiac function and the return function, the latter defined by the stressed blood volume, of which the bulk is in the small venules and veins [16]. As such, the CO response to phenylephrine is very dependent on the starting condition of the return function: if the patient is volume replete, with good reserves in unstressed volume and minimal initial tone in the veins draining the compliant region, phenylephrine can recruit unstressed volume into stressed volume by contractions of the smooth muscles in the walls of the vessels of the compliant part of the circulation, increasing the venous elastic recoil pressure. When this effect is greater than the increase in venous resistance, this will result in increased venous return and $\mathrm{CO}$ [16].

Figure 3 and Table 1 show that $90 \mathrm{~s}$ after an initial parallel increase in MAP and SVR following phenylephrine administration, the SVR curve started to decrease steeply, while the MAP curve drops more slowly. This dissociation indicates a second phenomenon increasing the blood pressure independently of the vascular resistance system. Because $\mathrm{MAP} \sim \mathrm{CO} \times \mathrm{SVR}$, an increase in venous return offers an additional contribution to the effect of phenylephrine on the MAP. The onset of this effect-about $90 \mathrm{~s}$ following the initial increase in MAP_corresponds to the expected time to reach a significant concentration of phenylephrine in the venous capacitance vessels. Remarkably, at $\mathrm{T}_{10}$, the SVR is not significantly higher compared to $\mathrm{T}_{-1}$, while MAP and CO are still 24 and 20\% higher, respectively (Table 1).

Next, the evolution of CVP-derived from the central venous catheter-also demonstrates a centralisation of venous blood, resulting in increased right ventricular preload, reflecting the postulated effects of phenylephrine on capacitance vessels. Even more, despite an increase in (left ventricular) $\mathrm{CO}$ - which depletes blood from the venous side-there is a persistent increase in CVP, implying that a higher cardiac preload is the primary driving force of the increased CO. This is also reflected in the steep increase in $\mathrm{SV}$ from 55 to $70 \mathrm{~mL}$ between $\mathrm{T}_{-1}$ and $\mathrm{T}_{5}$, despite the increase in systemic afterload, with no significant change in HR (Table 1). As surrogate measures of cardiac preload dependency, PPV-determined from the systolic and diastolic blood pressure measurements through the ventilation cycle-and SVV also distinctly drop. While PPV is not strictly a measure of preload, its decrease indicates a rightshift of the heart on the Frank-Starling relationship and thus a transition from preload dependence to fluid unresponsiveness without fluid administration [6]. Since all patients were preload dependent, no comparison in hemodynamic effects of phenylephrine between preload-dependent and preloadindependent states was possible.

As a separate independent measure, the evolution of the $\mathrm{EtCO}_{2}$ - measured by absorption spectrometry-indicates an increase in CO following phenylephrine [17, 18]. The significant average(SD) increase in $\mathrm{EtCO}_{2}$ from $38(4)$ to 42(5) $\mathrm{mmHg}$ during stable ventilatory settings and invariant $\mathrm{HR}$ also indicates an increase in $\mathrm{CO}$ owing to increased cardiac preload.

An important pharmacological consideration is the rather long biological half-life of phenylephrine of 2-3 h. While clinical experience based on the evolution of the MAP following phenylephrine administration gives the impression 


\section{Hemodynamic changes after phenylephrine}

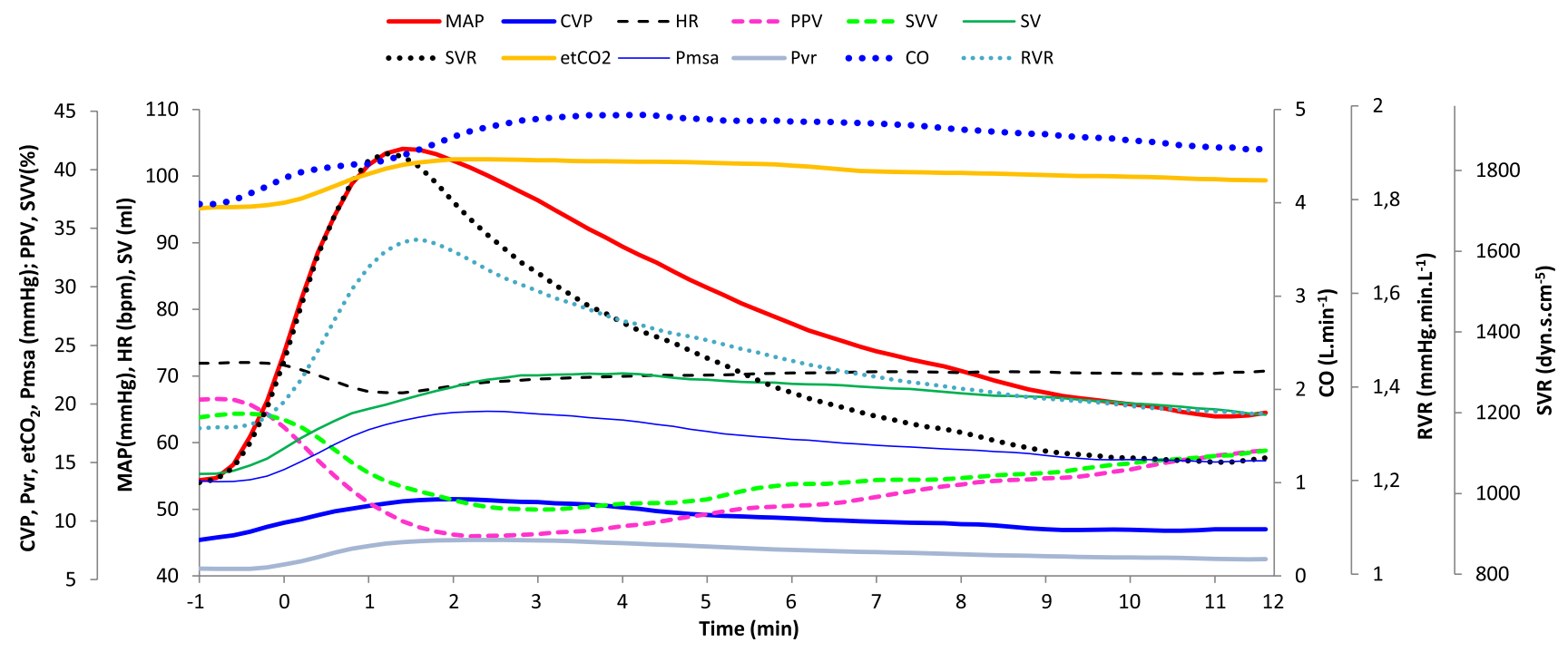

Fig. 3 The course of the hemodynamic variables after administration of phenylephrine. The MAP, CVP, HR, PPV, SVV, SV, SVR, end-tidal $\mathrm{CO}_{2}$-concentration $\left(\mathrm{EtCO}_{2}\right)$, mean systemic filling pressure (Pmsa), $\mathrm{CO}$ and resistance to vascular return (RVR) are shown.
The graphs are the averages of the individual patient measurements, synchronized at the moment $\left(\mathrm{T}_{0}\right)$ of $10 \mathrm{mmHg}$ increase in MAP after phenylephrine administration
Table 1 Evolution of the hemodynamic variables

\begin{tabular}{|c|c|c|c|c|c|}
\hline & $\mathrm{T}_{-1}$ & $\mathrm{~T}_{5}$ & $\mathrm{~T}_{10}$ & $\Delta\left(\mathrm{T}_{5}-\mathrm{T}_{-1}\right)$ & $\Delta\left(\mathrm{T}_{10}-\mathrm{T}_{-1}\right)$ \\
\hline MAP (mmHg) & $54(8)$ & $88(16)^{*}$ & $67(12)^{*}$ & $34(12 ; 64)$ & $13(2 ; 24)$ \\
\hline HR (bpm) & $72(10)$ & $70(12)$ & $70(11)$ & $-1(-12 ; 7)$ & $-2(-9 ; 5)$ \\
\hline $\mathrm{EtCO}_{2}(\mathrm{mmHg})$ & $38(4)$ & $42(5)^{*}$ & $40(5)^{*}$ & $3(0 ; 7)$ & $2(0 ; 6)$ \\
\hline CVP $(\mathrm{mmHg})$ & $8(5)$ & $11(6)^{*}$ & $9(5)$ & $2(-1 ; 9)$ & $0(-2 ; 5)$ \\
\hline $\mathrm{CO}\left(\mathrm{L} \mathrm{min} \min ^{-1}\right)$ & $3.92(0.87)$ & $4.94(1.2)^{*}$ & $4.71(1.23)^{*}$ & $0.9(-0.08 ; 2.57)$ & $0.55(-0.31 ; 2.25)$ \\
\hline $\mathrm{SV}(\mathrm{mL})$ & $55(10)$ & $70(14)^{*}$ & $67(14)^{*}$ & $17(3 ; 30)$ & $9(-1 ; 32)$ \\
\hline PPV (\%) & $20(7)$ & $9(5)^{*}$ & $13(5)^{*}$ & $-11(-21 ;-2)$ & $-6(-17 ;-2)$ \\
\hline $\operatorname{SVV}(\%)$ & $19(3)$ & $11(6)^{*}$ & $14(6)^{*}$ & $-8.5(-15 ; 3)$ & $-6(-11 ; 1)$ \\
\hline $\operatorname{SVR}\left(\right.$ dyn s cm $\mathrm{cm}^{-5}$ ) & $1035(305)$ & $1421(499)^{*}$ & $1103(350)$ & $370(80 ; 930)$ & $80(-110 ; 360)$ \\
\hline Pmsa (mmHg) & $13(4)$ & $18(5)^{*}$ & $15(5)^{*}$ & $4(0 ; 12)$ & $1(-1 ; 7)$ \\
\hline $\operatorname{RVR}\left(\operatorname{mmHg} \min \mathrm{L}^{-1}\right)$ & $1.33(0.34)$ & $1.54(0.45)^{*}$ & $1.38(0.38)^{*}$ & $0.09(0 ; 0.47)$ & $0.02(-0.06 ; 0.16)$ \\
\hline Pvr (mmHg) & $4.97(0.84)$ & $7.02(1.24)^{*}$ & $5.97(1.04)^{*}$ & $1.79(0 ; 3.81)$ & $0.84(-0.13 ; 2.01)$ \\
\hline
\end{tabular}

Mean(SD) evolution of the hemodynamic variables: before administration of phenylephrine $\left(\mathrm{T}_{-1}\right)$ and $5\left(\mathrm{~T}_{5}\right)$ and $10 \mathrm{~min}\left(\mathrm{~T}_{10}\right)$ after $10 \%$ increase in MAP. $* \mathrm{P}<0.05$ versus $\mathrm{T}_{-1}$. Median(range) changes in the hemodynamic variables between $\mathrm{T}_{-1}$ and $\mathrm{T}_{5}$, and between $\mathrm{T}_{-1}$ and $\mathrm{T}_{10}$

$M A P$ mean arterial pressure, $H R$ heart rate, $E t C \mathrm{O}_{2}$ end-tidal $\mathrm{CO}_{2}$ concentration, $C V P$ central venous pressure, $C O$ cardiac output, $S V$ stroke volume, $P P V$ pulse pressure variation, $S V V$ stroke volume variation, $S V R$ systemic vascular resistance, Pmsa mean systemic filling pressure, $R V R$ resistance to vascular return, $P v r$ pressure for venous return of a very short half-life, the fast decline of the MAP after the initial increase merely reflects the redistribution of the phenylephrine, but not its elimination. The long biological half-life of phenylephrine permits to reach a clinically significant plasma concentration in the capacitance vessels. While norepinephrine also has a potent $\alpha$-mimetic effect, it has a biological half-life of only $2-6 \min [19,20]$. Because of the fast enzymatic degradation of norepinephrine [20], the concentration in the capacitance vessels remains much lowerremind that the splanchnic capacitance vessels only receive a relatively small fraction of the $\mathrm{CO}$, while harbouring $25 \%$ of the total blood volume [21]. Given these pharmacokinetic differences, the ratio of the effects on $\mathrm{CO}$ and MAP will arguably be more balanced following phenylephrine 
compared to norepinephrine administration. This should, however, be substantiated in further research.

This emphasizes the physiological complexity determining the ultimate effect of the used vasopressor on the CO: (1) the balance of $\alpha$ - and $\beta$-adrenoceptor effects, (2) the pharmacokinetic properties of the vasoactive molecule, and (3) the preload dependency of the patient.

Our study has several limitations: firstly, no echocardiographic measurements of diastolic right and left volumes were performed to assess the preload effects of phenylephrine. The used ProAQT/Pulsioflex device is yet to be formally validated as sufficiently accurate to measure the absolute values of the variables of interest. Although calibrated devices offer more accurate absolute values, the relative changes to baseline values can be acceptably described by pulse wave contour analysis technology for assessing trend changes within a moderate range of acceptable physiological values. Additionally, the accuracy of the calculated SV based on wave-contour analysis may have been affected by the change in arterial elastance following phenylephrine administration [22]. The evolution of the CO, however, is only one of several variables indicating an increase in cardiac filling due to phenylephrine. The particular advantage of this device based on fast-reacting algorithms is its high temporal resolution, averaging 4 "sliding" intervals of $7.5 \mathrm{~s}$, which results in complete recalculation within $30 \mathrm{~s}$ [23]. Secondly, the patients were rather volume dependent due to the combination of vasoplegia by epidural analgesia and hypovolemia by bowel preparation, which may explain the distinct effects observed in this study, compared to other reports. Thirdly, atropine was administered at the start of anesthesia to attenuate the negative effects on the MAP and CO after induction with TIVA [24]. This doesn't influence the effects of phenylephrine on the venous return, but it probably blunts the reflex bradycardia induced by phenylephrine. While this is beneficial to more clearly demonstrate the effects on the venous return and global hemodynamics, it may narrow the external validity of its beneficial effects on the CO. Fourthly, Pmsa and Pvr values were derived mathematically and therefore, coupled with CO. Ideally, these values should have been assessed independently of CO. In our study, this was not done because the inspiratory hold manoeuvres to measure Pmsa would have unavoidably disturbed the accuracy of the primary research variables. The algorithm to calculate Pmsa and Pvr was validated previously [25]. Finally, no radiographic confirmation of the tip of the central venous catheter was performed at the time of CVP measurement. While this would arguably have minor influence on the calculated changes to baseline values of the studied variables, it may have affected the accuracy of the CVP measurements.

With respect to the described limitations, the current results must be interpreted within the constraints of potential shortcomings. Neither preload, contractility nor afterload were directly measured by the pulse contour technology. While the observed results suggest an improvement of venous return by virtue of phenylephrine administration, further research relying on direct measurements, like echography or thermodilution will be needed to fully describe the changes in cardiac preload, contractility and afterload in these clinical conditions.

In this study, the evolution of the hemodynamic variables was investigated after injection of a single dose of phenylephrine. This strategy was selected in order to most reliably depict the chronology of the hemodynamic changes. Additional research investigating the hemodynamic effects of phenylephrine in different baseline preload states may further elucidate the preload-dependency of these compound effects. The effects during continuous infusion on the investigated variables will have to be precisely determined, as well as comparison with the effects of alternative vasopressors. Ideally, an independent measure of $\mathrm{CO}$, such as ultrasound or thermodilution should have been used to confirm the observed evolution of waveform-derived variables.

\subsection{Clinical implications}

The prospect to optimize cardiac preload with considerably less fluid administration offers significant clinical advantages but adds complexity due to differences in pharmacokinetics and patient characteristics. Excessive administration of vasopressors may jeopardize organ perfusion, which underlines the importance of advanced hemodynamic monitoring to assess the evolution of the hemodynamic variables for the guidance of the hemodynamic management. In summary, in preload-dependent patients with low SVR, vasopressors could be preferable, while in preload-dependent patients with high SVR, volume administration would be a better choice. Meticulous trend assessment of different indices of $\mathrm{CO}$, volume responsiveness and SVR is imperative to individualize the optimal drug dose to maximise centralisation of blood while avoiding harmful effects on cardiac afterload and organ perfusion.

\section{Conclusions}

This study indicates that in preload-dependent patients, phenylephrine increases the $\mathrm{CO}$ by virtue of an increase in cardiac filling. This is manifested by several distinct hemodynamic indices of $\mathrm{CO}$ and venous return, namely the dissociation of MAP and SVR, CVP, PPV, CO, and $\mathrm{EtCO}_{2}$, in addition to the derived variables SV, SVV, Pmsa, Pvr and RVR.

Funding This study was solely supported by departmental funding. 


\section{Compliance with ethical standards}

Conflict of interest Thomas W.L. Scheeren received honoraria for consulting and lecturing from Edwards Lifesciences and from Masimo Inc. (Irvine, CA, USA). TWLS received honoraria from Pulsion Medical Systems SE for giving lectures. TWLS is associate editor of the Journal of Clinical Monitoring and Computing, but has no role in the handling of this paper. For the remaining authors none were declared.

Ethical approval All procedures performed in studies involving human participants were in accordance with the ethical standards of the institutional and/or national research committee and with the 1964 Helsinki declaration and its later amendments or comparable ethical standards.

Informed consent Informed consent was obtained from all individual participants included in the study.

Open Access This article is distributed under the terms of the Creative Commons Attribution 4.0 International License (http://creativeco mmons.org/licenses/by/4.0/), which permits unrestricted use, distribution, and reproduction in any medium, provided you give appropriate credit to the original author(s) and the source, provide a link to the Creative Commons license, and indicate if changes were made.

\section{References}

1. Shoemaker WC, Appel PL, Kram HB. Hemodynamic and oxygen transport responses in survivors and nonsurvivors of high-risk surgery. Crit Care Med. 1993;21:977-90.

2. Benes J, Giglio M, Brienza N, Michard F. The effects of goaldirected fluid therapy based on dynamic parameters on postsurgical outcome: a meta-analysis of randomized controlled trials. Crit Care. 2014;18:584.

3. Thiele RH, Nemergut EC, Lunch $\mathrm{C}$ 3rd. The clinical implications of isolated alpha(1) adrenergic stimulation. Anesth Analg. 2011;113:197-304.

4. Persichini R, Silva S, Teboul JL, Jozwiak M, Chemla D, Richard $\mathrm{C}$, Monnet X. Effects of norepinephrine on mean systemic pressure and venous return in human septic shock. Crit Care Med. 2012;40:3146-53.

5. Hamzaoui O, Georger JF, Monnet X, Ksouri H, Maizel J, Richard C, Teboul JL. Early administration of norepinephrine increases cardiac preload and cardiac output in septic patients with lifethreatening hypotension. Crit Care. 2010;14:R142.

6. Rebet O, Andremont O, Gérard JL, Fellahi JL, Hanouz JL, Fischer MO. Preload dependency determines the effects of phenylephrine on cardiac output in anaesthetised patients. A prospective observational study. Eur J Anaesthesiol. 2016;33:638-644.

7. Cannesson M, Zhongping J, Chen G, Vu TQ, Hatib F. Effects of phenylephrine on cardiac output and venous return depend on the position of the heart on the Frank-Starling relationship. J Appl Physiol. 2012;113:281-9.

8. Cecconi M, Aya HD, Geisen M, Ebm C, Fletcher N, Grounds RM, Rhodes A. Changes in the mean systemic filling pressure during a fluid challenge in postsurgical intensive care patients. Intensive Care Med. 2013;39:1299-305.
9. Faul F, Erdfelder E, Buchner A, Lang AG. Statistical power analyses using $\mathrm{G}^{*}$ Power 3.1: tests for correlation and regression analyses. Behav Res Methods. 2009;41:1149-60.

10. Zimmerman J, Cahalan M. Vasopressors and inotropes. In: Hemmings HC Jr, Egan TD, editors. Pharmacology and physiology for anaesthesia-foundation and clinical application. Philadelphia: Elsevier Saunders; 2013. pp. 390-404.

11. Schwarte LA, Scheeren TWL, Lorenz C, De Bruyne F, Fournell A. Moderate increase in intraabdominal pressure attenuates gastric mucosal oxygen saturation in patients undergoing laparoscopy. Anesthesiology 2004;100:1081-7.

12. Voldby AW, Brandstrup B. Fluid therapy in the perioperative setting-a clinical review. J Intensive Care. 2016;4:27.

13. Shin CH, Long DR, McLean D, et al. Effects of intraoperative fluid management on postoperative outcomes: a hospital registry study. Ann Surg. 2017. https://doi.org/10.1097/SLA.0000000000 002220 [Epub ahead of print].

14. Aga Z, Machina M, McCluskey SA. Greater intravenous fluid volumes are associated with prolonged recovery after colorectal surgery: a retrospective cohort study. Br J Anaesth. 2016;116:804-10.

15. Thiele RH, Nemergut EC, Lynch C 3rd. The physiologic implications of isolated alpha(1) adrenergic stimulation. Anesth Analg. 2011;113:284-296.

16. Magder S. Phenylephrine and tangible bias. Anesth Analg. 2011;113:211-3.

17. Lakhal K, Nay MA, Kamel T, Lortat-Jacob B, Ehrmann S, Rozec $\mathrm{B}$, Boulain T. Change in end-tidal carbon dioxide outperforms other surrogates for change in cardiac output during fluid challenge. Br J Anaesth. 2017;118:355-62.

18. Breen PH. How do changes in exhaled $\mathrm{CO}_{2}$ measure changes in cardiac output? A numerical analysis model. J Clin Monit Comput. 2010;6:413-9.

19. Hengstmann JH, Goronzy J. Pharmacokinetics of 3H-phenylephrine in man. Eur J Clin Pharmacol. 1982;21:335-41.

20. Beloeil H, Mazoit JX, Benhamou D, Duranteau J. Norepinephrine kinetics and dynamics in septic shock and trauma patients. Br J Anaesth. 2005;95:782-8.

21. Gelman S, Mushlin PS. Catecholamine-induced changes in the splanchnic circulation affecting systemic hemodynamics. Anesthesiology 2004;100:434-9.

22. Biais M, Mazocky E, Stecken L, Pereira B, Sesay M, Roullet S, Quinart A, Sztark F. Impact of systemic vascular resistance on the accuracy of the Pulsioflex device. Anesth Analg. 2017;124:487-93.

23. Umgelter A, Schmid RM, Huber W. Questionable design to validate the ProAQT/Pulsioflex device. Anesth Analg. 2017;125:1417-20.

24. Poterman M, Scheeren TW, van der Velde MI, Buisman PL, Allaert S, Struys MM, Kalmar AF. Prophylactic atropine administration attenuates the negative haemodynamic effects of induction of anaesthesia with propofol and high-dose remifentanil: a randomised controlled trial. Eur J Anaesthesiol. 2017;34:695-701.

25. Maas JJ, Pinsky MR, Geerts BF, de Wilde RB, Jansen JR. Estimation of mean systemic filling pressure in postoperative cardiac surgery patients with three methods. Intensive Care Med. 2012;38:1452-60. 